\title{
L'image reliante de Michel \\ Maffesoli
}

\section{RESUMÉ}

Le texte mostre les différents moyens à partir de l'oeuvre de Michel Maffesoli pour compreendre l'image.

\section{RESUMO}

O texto mostra os diferentes meios para se compreender a imagem a partir da obra de Michel Maffesoli.

\section{PALAVRAS-ChaVe (MOT-CLÉS)}

- Michel Maffesoli

- Imagem (Image)

- Vivência Social (Vécu Social)

\section{Federico Casalegno}

Chercheur au MIT/CeaQ

\section{Stéphane Hugon}

SI L'ON DIT PARFOIS DE Michel Maffesoli qu'il est un penseur de l'image, il fait ajouter qu'il est aussi penseur par l'image. A la fois substance et méthode, celle-ci se glisse et prolifère dans son œuvre, tout comme elle est présente dans son enseignement. C'est en cela que Michel Maffesoli donne à penser, plus qu'il ne livre des concepts; ses images donnent à croître, et comprennent une dimension d'initiation, plus que de pédagogie.

L'image comme mesocosme, comme élément interstitiel entre le macrocosme et le microcosme, qui en même temps relie et déclenche la compréhension de la réalité quotidienne, du vécu, du social.

Il travaille avec les mots, et travaille les mots eux-mêmes, de manière à ce qu'ils ne tarissent pas de sens et ne se figent pas dans leurs significations. Les notions et les images ont ceci en eux de dynamique, qu'ils n'enferment pas mais développent et prolongent le sens au-delà du dit. L'idée se propage et peut résonner dans d'autres lieux - terrains, imaginaires -, ainsi les notions et images maffesoliennes ne limitent pas ni ne closent, elles renvoient, réaniment, dévoilent, remémorent. L'évocation d'images, de mots, leurs étymologies, Michel Maffesoli les pratique, et, pour ainsi dire les travaille au corps. Retrouvant ainsi une indication de Gaston Bachelard ( $\mathrm{Ce}$ qui nous trompe, c'est que le même mot à la fois désigne et explique. »), Michel Maffesoli pratique ainsi une pensée qui déborde, qui s'égrène, et qu'il dira séminale.

S'il s'est largement expliqué sur ce retour à l'image - à sa genèse, à sa dimension heuristique -, et sur le contexte culturel qui lui donne corps, il faut néanmoins redire la justesse de son travail, pour une lecture de notre culture contemporaine en ce qu'elle est à la fois fascinée par l'image, et pourtant comme encore médusée par le pouvoir du texte. Il y a probablement ici l'expression 
d'un curieux paradoxe, celui qui voit notre culture moderne, qui a longtemps puisé sa tradition dans le rejet des images et des formes, ou tout au moins avec une grande suspicion pour tout ce qui suscite des glissements interprétatifs non binaires, les analogies, les métaphores, et qui finalement va permettre, dans son époque tardive, l'émergence et la circulation technique d'images sous ses formes variées. Alors que notre épistémologie se fonde sur le texte, dans sa linéarité, et dans sa logique aristotélicienne, il semblerait que resurgisse, sous différentes formes, et à différents degrés, ces images, envahissantes, virales, et qui pour le sociologue pourraient constituer un matériau de premier choix.

Gilbert Durand souligne bien ce paradoxe; nous vivons une époque de grandes productions techniques et technologiques, mais il devient difficile d'accepter les manifestations de ce vitalisme polymorphe, effervescent et éclaté : «Il nous faut préciser ce paradoxe d'une civilisation, la nôtre, qui, d'un côté, a apporté au monde les techniques, sans cesse en progrès, de la production, de la reproduction, de la communication, et de l'autre, du côté de sa philosophie fondamentale, a fait preuve d'une méfiance iconoclaste endémique.»

Et c'est dans ce contexte que la pensée audacieuse de Michel Maffesoli saisi le vitalisme polymorphe et éclatée se manifestant dans le cyberespace. Sa pensée prend donc une dimension particulière lorsqu'on la convie à une étude des médias, et des communications en réseau pour lesquelles le scintillement des écrans de surfaces empiète sur le texte ou sur des logiques plus linéaires.

Rappelons simplement combien les études des technologies de communication sont encore très fixées sur le message, dans son contenu sémantique, sur la fonction transitive des medias, et sur l'individualisation de l'émetteur et du récepteur. Il convient pourtant aujourd'hui, de repérer la saturation d'une expertise qui reste par trop substantialiste, et causaliste.
Comme si les études des réseaux étaient encore modelées par une posture « cybernétique »: la communication en des termes organisationnels et, plus encore, la communication liée à l'idée de commande. Dans la perspective cybernétique l'information communiquée devient programme : elle constitue des "instructions » ou «ordres» qui déclenchent, inhibent, coordonnent les opérations. L'élément de la communication est subordonné à celui de la commande. Mais c'est également dans les sciences sociales que cette posture se voit transférée. Bon nombre d'observateurs délaissent ainsi malgré eux la communication au profit de la seule information, réduite à un objet, et un flux. Lorsque la communication se fait technique, elle rejoint ainsi l'objet fétiche de notre culture moderne. Aboutissement de la séparation et de l'arraisonnement, la technique moderne converge au fonctionnel et à l'utilitaire. Plus qu'outil, elle devient culture, dans le sens où elle induit une spécialisation, une standardisation, une temporalité, et une fonctionnalisation sociale. Et qui conduit à ce que Max Weber appelle le «désenchantement du monde».

Avec Michel Maffesoli nous comprenons l'importance du contenant sur le contenu, forme de communication vide, sans message binaire clairement déchiffrable, mais qui donne du sens à l'échange.

Cette communication tactile nous permet de participer à un « nous communautaire », où la force d'agrégation repose, plus que sur le contenu du message échangé, mais sur le contact de proximité qu'elle induit. Par la communication je rentre en contact avec l'autre, je fusionne avec le grand "Autre ", et ce sont ces réseaux de communication qui font resurgir des formes d'oralité. En ce sens, on est plus attentif au contenant qui sert de toile de fond, qui crée ambiance et par là unit.

Or, l'on ne pouvait se résoudre à croire que tant d'appétence pour l'image, les connexions, les réseaux, ne s'expliquerait par une curieuse addiction forcée ou contrainte. 
Ici, les travaux de Michel Maffesoli nous permettent de comprendre plus profondément la nature sociale de ces phénomènes grégaires. La notion de socialité, ainsi que la figure de l'orgiaque qui en est issue, nous convient ainsi à une approche des manifestations par le contact, par le toucher, et pour ainsi dire les à-côtés de l'information, par son événement social.

Ces notions montrent comment les phénomènes sociaux contemporains réinvestissent les objets techniques afin de leur donner un sens différent, et de permettre les moments de partage, d'effervescence, autour d'échange d'image, ou de micro événements qui structurent le quotidien.

Il s'agit là d'une défonctionnalisation de la technique, dans le sens où elle n'est plus employée pour servir une maîtrise ou une domination de l'environnement - humain, naturel, physique -, mais bien pour célébrer une dépense au sens que lui donne Georges Bataille. La dimension sociale, relationnelle, rituelle, se passe pour ainsi dire de contenu. Celui-ci devenu prétexte, peut permettre l'agrégation, qu'il soit un feuilleton à la télévision, une image sur l'Internet, un texte très court sur le téléphone. La technique se fait alors le creuset d'une perte, proche de ce que Asger Jorn appelait l'inutilisation des objets.

Aborder ainsi les techniques d'images, c'est permettre de retrouver la capacité agrégative des technologies de réseau ; c'est également se rendre attentif à la manière dont les technologies qui privilégient le contact pur, la réactivité, la viscosité dira Michel Maffesoli, sont aujourd'hui sollicitées, ainsi que tous les outils qui permettent les regroupements éphémères. La relation sociale en ligne - fût-ce par le biais de l'image - peut apparaître pour ce qu'elle est, c'est-à-dire portée par l'événementialité de son jeu et de sa ritualisation à l'infini. Les regroupements, les relations et les moments d'effervescence relationnelle peuvent ainsi apparaître, sans objet, sans but, et dans la poétique du partage d'un moment unique, autoréférenciel et souvent de courte durée.
Et qui rendent ainsi énigmatique, ou caduque, la question du message, du manifeste ou du programme. La rupture - même dans la lenteur - du mode d'analyse est donc de taille. Les technologies de communication inversent nos manières de voir. La socialité renvoie à une forme de relation sociale qui n'est plus fondée sur un projet, et qui utiliserait la technique comme moyen, mais bien plutôt comme un espace de lieu et de temps, où va s'effectuer une communion.

Cette communion a des prétextes parfois très rationnels. Mais ils n'apparaissent que comme des déclencheurs. L'essentiel de la relation et de la fusion sociale se fait par le partage païen, et sur les petites imitations (Gabriel Tarde).

Michel Maffesoli a ainsi pensé, dans un même mouvement, la socialité et la technique, en faisant rentrer en synergie les éléments dionysiaques liés à la dépense, l'effervescence et l'empathie qui se consume au fil des réseaux avec l'outil technique. Il souligne cette fonction rituelle de la communication où les fonctions de commémoration, d'anamnèse, se passent de la recherche de contenu, pour se focaliser sur le geste et l'événement . 